\title{
LOWER BOUNDS FOR THE ZEROS OF BESSEL FUNCTIONS
}

\author{
ROGER C. MCCANN
}

Abstract. Let $j_{p, n}$ denote the $n$th positive zero of $J_{p}, p>0$. Then

$$
j_{p, n}>\left(j_{0, n}^{2}+p^{2}\right)^{1 / 2} \text {. }
$$

We begin by considering the eigenvalue problem

$$
\begin{gathered}
-\left(x y^{\prime}\right)^{\prime}+x^{-1} y=\lambda^{2} x^{2 p-1} y, \quad \lambda, p>0, \\
y(a)=y(1)=0, \quad 0<a<1 .
\end{gathered}
$$

For simplicity of notation we will set $q=p^{-1}$. It is easily verified that the general solution of (1) is

$$
y(x)=C_{1} J_{q}\left(\lambda q x^{1 / q}\right)+C_{2} Y_{q}\left(\lambda q x^{1 / q}\right)
$$

and that the eigenvalues are given by

$$
J_{q}(\lambda q) Y_{q}\left(\lambda q a^{1 / q}\right)-J_{q}\left(\lambda q a^{1 / q}\right) Y_{q}(\lambda q)=0 .
$$

If $z_{n}(a, r)$ denotes the $n$th positive zero of $J_{r}(z) Y_{r}\left(z a^{1 / q}\right)-J_{r}\left(z a^{1 / q}\right) Y_{r}(z)=$ 0 , then the $n$th eigenvalue, $\lambda_{n}^{2}(a)$, of (1), (2) is given by

$$
\lambda_{n}^{2}(a)=\left(z_{n}(a, q) / q\right)^{2} .
$$

Let $j_{r, n}$ denote the $n$th positive zero of $J_{r}$. On p. 38 of [4] it is shown that $z_{n}(a, r) \rightarrow j_{r, n}$ as $a \rightarrow 0^{+}$whenever $r$ is a positive integer. The restriction on $r$ is extrinsic so that

$$
\lim _{a \rightarrow 0^{+}} z_{n}(a, r)=j_{r, n}, \quad r \geqslant 0 .
$$

Let $R[p, y]$ denote the Rayleigh quotient

$$
R[p, y]=\int_{a}^{1}\left(-\left(x y^{\prime}\right)^{\prime}+x^{-1} y\right) y d x / \int_{a}^{1} x^{2 p-1} y^{2} d x
$$

It is well known that the eigenvalues $\left\{\lambda_{n}^{2}(p)\right\}$ of (1), (2) can be obtained from the Rayleigh quotient [5]. Let $V$ denote the linear space of all functions in $C^{2}((a, 1))$ which satisfy the boundary conditions $(2)$. Then

$$
\lambda_{1}^{2}(p)=\min _{y \in V, y \neq 0} R[p, y] .
$$

Let $y_{1}, y_{2}, \ldots, y_{n}$ be $n$ functions in $V, A$ denote the subspace of $V$ spanned by $y_{1}, y_{2}, \ldots, y_{n}$ and $A^{\perp}$ denote the orthogonal complement of $A$ relative to $V$. Then

Received by the editors January 5, 1976 and, in revised form, September 13, 1976. AMS (MOS) subject classifications (1970). Primary 33A40. 


$$
\lambda_{n+1}^{2}(p)=\max _{A} \min _{y \in A^{\perp}, y \neq 0} R[p, y]
$$

where the maximum is taken over all sets of $n$ functions in $V$.

Whenever $p>0$ we have that $x^{2 p-1}<x^{-1}$ for all $x \in(0,1)$. Then

$$
R[p, y]=\frac{\int_{a}^{1}-\left(x y^{\prime}\right)^{\prime} y d x}{\int_{a}^{1} x^{2 p-1} y^{2} d x}+\frac{\int_{a}^{1} x^{-1} y^{2} d x}{\int_{a}^{1} x^{2 p-1} y^{2} d x} \geqslant Q[p, y]+1,
$$

where

$$
Q[p, y]=\int_{a}^{1}-\left(x y^{\prime}\right)^{\prime} y d x / \int_{a}^{1} x^{2 p-1} y^{2} d x
$$

is the Rayleigh quotient for the eigenvalue problem

$$
\begin{aligned}
-\left(x y^{\prime}\right)^{\prime} & =\mu^{2} x^{2 p-1} y, \\
y(a) & =y(1)=0,
\end{aligned}
$$

Equation (6) is equivalent to

$$
x^{2} y^{\prime \prime}+y x^{\prime}+\mu^{2} x^{2 p} y=0 .
$$

It is easily checked that the general solution of (8) and, hence, of (6) is (recall that $q=p^{-1}$ )

$$
y(x)=C_{1} J_{0}\left(\mu q x^{1 / q}\right)+C_{2} Y_{0}\left(\mu q x^{1 / q}\right)
$$

and that the eigenvalues are given by

$$
J_{0}(\mu q) Y_{0}\left(\mu q a^{1 / q}\right)-J_{0}\left(\mu q a^{1 / q}\right) Y_{0}(\mu q)=0 .
$$

In particular the $n$th eigenvalue, $\mu_{n}^{2}(a)$, of (6), (7) is given by

$$
\mu_{n}^{2}(a)=\left(z_{n}(a, 0) / q\right)^{2} \text {. }
$$

From (3), (5), and (9) we obtain

$$
\left(z_{n}(a, q) / q\right)^{2} \geqslant\left(z_{n}(a, 0) / q\right)^{2}+1 .
$$

If we now replace $q$ by $p$, let $a \rightarrow 0^{+}$in (10), and using (4) we find that $\left(j_{p, n} / p\right)^{2} \geqslant\left(j_{0, n} / q\right)^{2}+1$.

THEOREM. $j_{p, n} \geqslant\left(\left(j_{0, n}\right)^{2}+p^{2}\right)^{1 / 2}$ whenever $p \geqslant 0$.

COROLLARY. $j_{p, n}>\left(\left(n-\frac{1}{4}\right)^{2} \pi^{2}+p^{2}\right)^{1 / 2}$ whenever $p \geqslant 0$.

Proof. It is known (see [9, p. 489]) that the positive zeros of $J_{0}$ lie in the intervals $\left(m \pi+\frac{3}{4} \pi, m \pi+\frac{7}{8} \pi\right)$ for $m=0,1,2, \ldots$ Hence, $j_{0, n}>(n-1) \pi$ $+\frac{3}{4} \pi=\left(n-\frac{1}{4}\right) \pi$. The desired result follows.

In [8] it is shown that

$$
j_{p, n}=p+a_{n} p^{1 / 3}+b_{n} p^{-1 / 3}+O\left(p^{-1}\right) \quad(n=1,2, \ldots),
$$

where $a_{n}$ and $b_{n}$ are independent of $p$. Hence,

$$
j_{p, n}^{2}=p^{2}+c_{n} p^{4 / 3}+O\left(p^{2 / 3}\right) \quad(n=1,2, \ldots),
$$

where $c_{n}$ is independent of $p$. This shows that the second term of the lower 
bound for $j_{p, n}$ given in the Theorem is of the wrong order. Other asymptotic expansions for $j_{p, n}$ may be found in [1], [2], and [6].

In [3] it is shown that for $0 \leqslant p \leqslant \frac{1}{2}$

$$
p \pi / 2+\left(n-\frac{1}{4}\right) \pi \leqslant j_{p, n} .
$$

For $p=0$ the result of the Theorem is exact, while the expression in (11) has a strict inequality. Hence, our result is stronger than (11) whenever $p$ is sufficiently small. However, when $p=\frac{1}{2}$, the result in (11) is exact. Hence, for $0 \leqslant p \leqslant \frac{1}{2}$ neither result implies the other. It should be emphasized that the Theorem is valid for all $p \geqslant 0$, while (11) is valid only for $0 \leqslant p \leqslant \frac{1}{2}$.

I would like to thank the referee for his helpful suggestions and for bringing [2], [3], and [4] to my attention.

\section{REFERENCES}

1. T.H. Boyer, Concerning the zeros of some functions related to Bessel functions, J. Mathematical Phys. 10 (1969), 1729-1744. MR 41 \#2074.

2. H.W. Hethcote, Error bounds for asymptotic approximations of zeros of transcendental functions, SIAM J. Math Anal. 1 (1970), 147-152. MR 42 \# 744.

3. __ Bounds for zeros of some special functions, Proc. Amer. Math. Soc. 25 (1970), 72-74. MR 41 \#569.

4. M. Kline, Some Bessel equations and their application to guide and cavity theory, J. Mathematical Phys. 27 (1948), 37-48. MR 9, 431.

5. S.G. Mikhlin, Variational methods in mathematical physics, GITTL, Moscow, 1957; English transl., Macmillan, New York, 1964. MR 22 \# 1981; 30 \#2712.

6. F.W.J. Olver, The asymptotic expansion of Bessel functions of large order, Philos. Trans. Roy. Soc. London Ser. A247 (1954), 328-368. MR 16, 696.

7. (Editor), Bessel functions. Part III, Roy. Soc. Math. Tables, Vol. 7, Cambridge Univ. Press, Cambridge, 1960.

8. F. Tricomi, Sulle funzioni di Bessel di ordine e argomento pressochèuguali, Atti. Accad. Sci. Torino Cl. Sci. Fis. Mat. Nat. 83 (1949), 3-20. MR 11, 594.

9. G.N. Watson, A treatise on the theory of Bessel functions, Cambridge Univ. Press, Cambridge, 1958.

Department of Mathematics, Case Western Reserve University, Cleveland, Ohio 44106 